\title{
Vehicle Speed Tracking Using Chassis Vibrations
}

\author{
Martin Lindfors, Gustaf Hendeby, Fredrik Gustafsson and Rickard Karlsson
}

\section{Linköping University Post Print}

\section{Tweet}

N.B.: When citing this work, cite the original article.

Original Publication:

Martin Lindfors, Gustaf Hendeby, Fredrik Gustafsson and Rickard Karlsson, Vehicle Speed Tracking Using Chassis Vibrations, 2016, Proceedings of the 2016 IEEE Intelligent Vehicles Symposium (IV), 214-219.

http://dx.doi.org/10.1109/IVS.2016.7535388

Copyright: Not Found

$\underline{\text { IEEE }}$

Postprint available at: Linköping University Electronic Press

http://urn.kb.se/resolve?urn=urn:nbn:se:liu:diva-129689 


\title{
Vehicle Speed Tracking Using Chassis Vibrations
}

\author{
Martin Lindfors, Gustaf Hendeby, Fredrik Gustafsson and Rickard Karlsson \\ Dept. of Electrical Engineering, Linköping University, Sweden \\ E-mail: martin.lindfors@liu.se, \{hendeby, fredrik, rickard\}@isy.liu.se.
}

\begin{abstract}
The speed of a wheeled vehicle is usually estimated using wheel speed sensors (WSS) or GPS. If these signals are unavailable, other methods must be used. We propose a novel approach exploiting the fact that vibrations from rotating axles, with fundamental frequency proportional to vehicle speed, are transmitted via the vehicle chassis. Using an accelerometer, these vibrations can be tracked to estimate vehicle speed while other sources of vibrations act as disturbances. A state-space model for the dynamics of the harmonics is presented and formulated such that there is a conditional linear-Gaussian substructure, enabling efficient Rao-Blackwellized methods. A variant of the Rao-Blackwellized point-mass filter is derived, significantly reducing computational complexity, and reducing the memory requirements from quadratic to linear in the number of grid points. It is applied to experimental data from the sensor cluster of a car and validated using the rotational frequency from wSS data. The proposed method shows improved performance and robustness in comparison to a Rao-Blackwellized particle filter implementation and a frequency spectrum maximization method.
\end{abstract}

\section{INTRODUCTION}

Many methods exist to determine the velocity of a vehicle. For wheeled vehicles such as passenger cars, the dominant method is to use wheel speed sensors (WSS) [1], which accurately measure the angular velocity of the wheels. This quantity is proportional to vehicle velocity but the proportionality constant, the wheel radius, is in general unknown. The method also requires installation of wSS sensors at the wheels, and the velocity estimation algorithm must be able to retrieve measurements from the sensors.

Alternative solutions for everyday vehicle applications include GPS Doppler measurements [2,3] or inertial navigation [4]. However, GPS access may be limited in tunnels, indoors environments and "urban canyons" [5]; and due to sensor imperfections, methods based on integration of accelerometer measurements yield large errors after short periods of time.

In this paper a new method is proposed that utilizes a property embedded in the accelerometer signals. It has been observed that the accelerometer signal contains a frequency component proportional to the speed of the vehicle (see Fig. 1,2), and by tracking it a proportional velocity estimate is obtained. This enables access to wheel angular velocity when direct speed measurements from WSS and GPS sensors are unavailable. Instead, we indirectly estimate the vehicle speed using vibrations.

Possible applications range from stand-alone road vehicle navigation systems in tunnels or urban environments where GPS satellites may be unavailable; to indoor vehicles such as small forklifts or pallet jacks where wsS are expensive

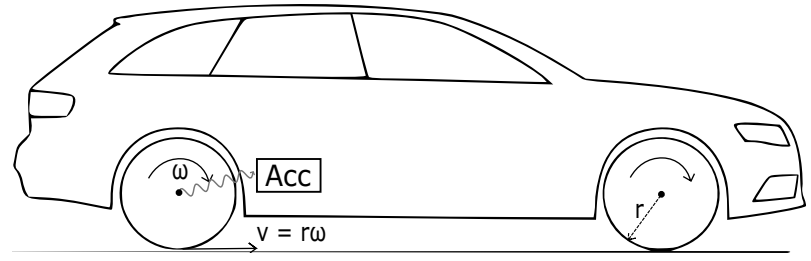

Fig. 1. Illustration of a vehicle equipped with an accelerometer (Acc). Vibrations (grey) arise from the wheel axles, and are transmitted to the vehicle chassis, where the accelerometer is mounted and can measure them. The idea is to estimate velocity by tracking the frequency of the vibrations, without access to the wSS.

or unfeasible, while an accelerometer is available in the employee's smartphone and can be attached to the vehicle.

Many different methods have been proposed to track the frequency or harmonics (pitch) of a signal. In this paper, the focus is on parametric Bayesian methods, in which the continuity of the fundamental frequency, or vehicle wheel speed, is easily incorporated.

In [6-8] the extended Kalman filter (EKF) is used to track the frequency of a sine in noise. The results of [9] indicate that the particle filter $(\mathrm{PF})$ is well suited for frequency tracking. For harmonics tracking, the authors of $[10,11]$ apply the Rao-Blackwellized particle filter (RBPF) [12-14] to the problem. Other, non-Bayesian methods to solve the harmonics estimation problem include methods based on maximizing power spectra [15], and ones using the crosscorrelation function [16]. These methods are popular in audio processing, where sample rates typically are higher.

In Fig. 2, an example of a spectrogram of the accelerometer signal is illustrated along with the ground truth angular frequency given by data from the wSS signal. As can be observed, the fundamental frequency, or first harmonic, is often clearly visible and coincides well with the wss value. Higher harmonics sometimes also appear, most clearly the second and third. Note that there are no visible harmonics which arise from the engine or gearbox for this vehicle. However, none of the harmonics are available at every point in time; the higher harmonics disappear around $t \approx 240 \mathrm{~s}$ and the first harmonic is very weak at $t \approx 265 \mathrm{~s}$. Keeping this in mind, it is clear that filtering algorithms applied to this type of data must be able to handle those various cases robustly. This motivates some kind of sequential processing which exploits the fact that vehicle velocity usually does not change abruptly.

In this paper, a method based on the Rao-Blackwellized 


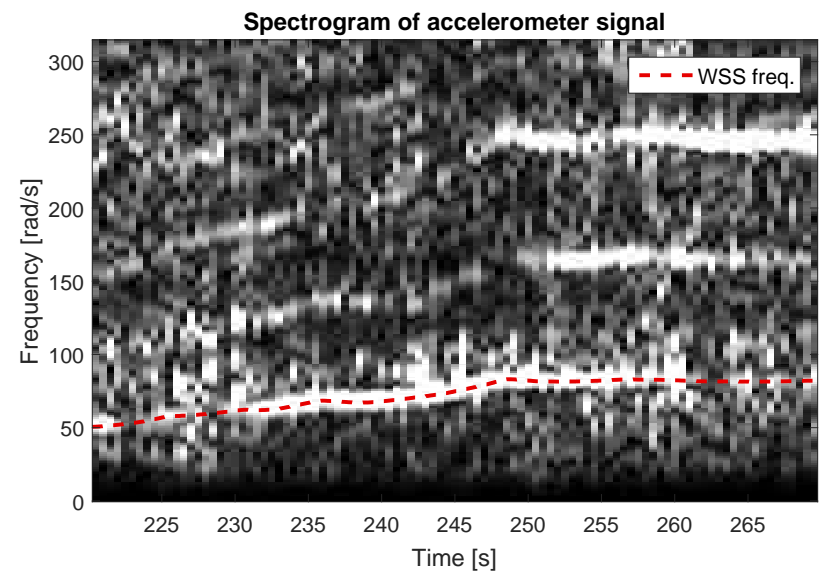

Fig. 2. Spectrogram of the high-pass filtered accelerometer signal, collected in a passenger car, with fundamental frequency given by the wsS. Higher harmonics can often be seen at integer multiples of the wss, but no harmonic is present at every point in time. Brighter shades correspond to higher energies.

point-mass filter (RBPMF) [17, 18] is proposed and compared to the RBPF and a power spectrum maximization method on experimental vehicle data. The RBPMF can handle many hypotheses, even if they are initially unlikely, and is robust to initial misdetections. Sec. II formulates the problem and proposes different models to approach the problem. Sec. III develops Bayesian filters utilizing the structure of the model. Sec. IV evaluates the methods on the experimental accelerometer data, and Sec. V concludes the paper.

\section{Problem Formulation And Modeling}

The filtered accelerometer measurements are denoted $y_{t}$. The signal $y_{t}$ contains harmonics where the first harmonic is given by the true wheel angular velocity; $\omega^{*}=v / r$, where $v$ is the absolute vehicle speed and $r$ the wheel radius. The higher harmonics are given by $k \omega^{*}$ for $k=2,3$. To estimate $\omega^{*}$, it is insufficient to simply maximize the discrete Fourier transform (DFT), since there are amplitude and frequency variations and the first harmonic sometimes is weak or disappears. One approach is to model the instantaneous amplitudes and phases (Sec. II-A), another to average over time and estimate the fundamental frequency by maximizing the combined energy of the harmonics (Sec. II-B).

\section{A. State-space Harmonics Model}

Consider the following nonlinear state-space model, with state $x_{t}$, measurement $y_{t}$, process noise $v_{t}$ and measurement noise $e_{t}$ :

$$
\begin{aligned}
x_{t+1} & =f\left(x_{t}\right)+v_{t}, \\
y_{t} & =h\left(x_{t}\right)+e_{t} .
\end{aligned}
$$

One way to represent a harmonic oscillation is given by the phasor representation, i.e., representing the instant phase and amplitude by a complex number. Let the instant phase and amplitude of the $m$ :th harmonic at $t$ be represented by the phasor $\alpha_{t}^{(m)}+i \beta_{t}^{(m)}$. Given a constant angular velocity $m \omega_{0}$, the phasor dynamics is given by

$$
\begin{aligned}
\alpha_{t}^{(m)}+i \beta_{t}^{(m)} & =e^{i m \omega_{0} T}\left(\alpha_{t-1}^{(m)}+i \beta_{t-1}^{(m)}\right) \\
& =e^{i m \omega_{0} t T}\left(\alpha_{0}^{(m)}+i \beta_{0}^{(m)}\right)
\end{aligned}
$$

yielding

$$
\alpha_{t}^{(m)}=\left|\alpha_{0}^{(m)}+\beta_{0}^{(m)}\right| \cos \left(m \omega_{0} t T+\arg \left(\alpha_{0}^{(m)}+i \beta_{0}^{(m)}\right)\right) .
$$

It is then clear that $\alpha_{t}^{(m)}$ is a sinusoid with phase offset, amplitude and angular velocity defined by $\alpha_{0}^{(m)}, \beta_{0}^{(m)}$ and $\omega_{0}$. A measurement equation for multiple harmonics is given by considering a sum of $M$ sinusoids $a_{t}^{(m)}$ corrupted by measurement noise,

$$
y_{t}=h\left(x_{t}\right)+e_{t}=\sum_{m=1}^{M} \alpha_{t}^{(m)}+e_{t} .
$$

Selecting the state vector $x_{t}$ as

$$
x_{t}=\left[\omega_{t}, \alpha_{t}^{(1)}, \alpha_{t}^{(2)}, \ldots, \alpha_{t}^{(M)}, \beta_{t}^{(1)}, \ldots, \beta_{t}^{(M)}\right]^{\top},
$$

we can implement the dynamics (2a) through

$$
\begin{aligned}
\omega_{t+1} & =\omega_{t}+\sqrt{T} v_{t}^{\omega}, \\
{\left[\begin{array}{c}
\alpha_{t+1}^{(m)} \\
\beta_{t+1}^{(m)}
\end{array}\right] } & =R\left(m \omega_{t} T\right)\left[\begin{array}{l}
\alpha_{t}^{(m)} \\
\beta_{t}^{(m)}
\end{array}\right]+\sqrt{T}\left[\begin{array}{c}
v_{t}^{\alpha^{(m)}} \\
v_{t}^{\beta^{(m)}}
\end{array}\right],
\end{aligned}
$$

where independent process noises $v_{t}^{\omega}, v_{t}^{\alpha^{(m)}}, v_{t}^{\beta^{(m)}}$ have been added to make the model adaptable to changes in frequency, amplitude, and phase offset. The matrix

$$
R(\psi)=\left[\begin{array}{cc}
\cos \psi & \sin \psi \\
-\sin \psi & \cos \psi
\end{array}\right]
$$

is the ordinary two-dimensional rotation matrix which implements the complex exponential multiplication in (2a). Thus, the model (4) is on the form (1). Furthermore, the model can be cast in a conditionally linear-Gaussian form (see Sec. IV-B), which makes it appropriate to use in conjunction with a certain class of Bayesian filtering methods.

\section{B. Subharmonic Summation}

A baseline spectrum-based method, subharmonic summation (SHS) [15], is used in comparison with the proposed methods. The idea of the method is that the magnitude spectrum contains peaks at multiples of the fundamentals, so that an estimate of the fundamental frequency can be computed as

$$
\hat{\omega}=\arg \max _{\omega} \sum_{k=1}^{(m)} c_{k}|Y(k \omega)|^{2},
$$

where $Y(\omega)$ is a possibly preprocessed frequency transform of the signal $y_{t}$ and $c_{k}$ are harmonics scales. SHS computes a smoothed magnitude spectrum of a signal, interpolates the frequency to a logarithmic scale, filters it in the frequency domain, and sums different translated, scaled spectra together. Subsequently the summed spectrum is maximized, and the estimated fundamental frequency is given by the frequency 
of the maximum. The main difference between the presented method in [15] and the method used in this paper is that the "peak enhancement" step is omitted since it appears counterproductive in this application.

\section{BAYESIAN FILTERING}

The objective of Bayesian estimation is to determine the distribution of an unknown state, $x_{t}$, at time $t$, given all measurements $\mathbb{Y}_{t}=\left\{y_{\tau}\right\}_{\tau=1}^{t}$ available up until time $t$.

\section{A. Recursive Bayesian Estimation}

A recursive Bayesian estimation formulation consists of solving the following time update and measurement update equations for the probability density functions (PDFs):

$$
\begin{aligned}
p\left(x_{t+1} \mid \mathbb{Y}_{t}\right) & =\int p\left(x_{t+1} \mid x_{t}\right) p\left(x_{t} \mid \mathbb{Y}_{t}\right) d x_{t} \\
p\left(x_{t} \mid \mathbb{Y}_{t}\right) & =\frac{p\left(y_{t} \mid x_{t}\right) p\left(x_{t} \mid \mathbb{Y}_{t-1}\right)}{\int p\left(y_{t} \mid x_{t}\right) p\left(x_{t} \mid \mathbb{Y}_{t-1}\right) d x_{t}} .
\end{aligned}
$$

The PF, [19, 20], provides a fundamental solution to many recursive Bayesian filtering problems, incorporating both nonlinear and non-Gaussian systems. This extends the classic optimal filtering theory developed for linear and Gaussian systems, where the optimal solution is given by the Kalman filter (KF) [21, 22]. For some problems direct numerical discretization of the Bayesian integrals might be an alternative, resulting in the point-mass filter (PMF), [23]. A review of Bayesian filtering algorithms can be found in [24].

\section{B. General Model for Rao-Blackwellized Methods}

If the model contains a conditionally linear Gaussian subsystem, e.g., the model structure (4), it is possible to utilize this in an efficient implementation usually referred to as a Rao-Blackwellized filter. Partition the state vector $x$ into two parts, $x=\left[\begin{array}{l}x^{p} \\ x^{k}\end{array}\right]$, so that it is possible to factorize the posterior distribution, $p\left(x_{t} \mid \mathbb{Y}_{t}\right)$, as

$$
p\left(\mathbb{X}_{t}^{p}, x_{t}^{k} \mid \mathbb{Y}_{t}\right)=p\left(x_{t}^{k} \mid \mathbb{X}_{t}^{p}, \mathbb{Y}_{t}\right) p\left(\mathbb{X}_{t}^{p} \mid \mathbb{Y}_{t}\right)
$$

If the partition is chosen such that the model is conditionally linear-Gaussian, i.e., if the term $p\left(x_{t}^{k} \mid \mathbb{X}_{t}^{p}, \mathbb{Y}_{t}\right)$ is linearGaussian, it can be optimally estimated using a KF. To obtain the second factor, it is necessary to apply nonlinear filtering techniques such as the PF or PMF.

Now consider the following general state-space model:

$$
\begin{aligned}
x_{t+1}^{p} & =f^{p}\left(x_{t}^{p}\right)+F^{p}\left(x_{t}^{p}\right) x_{t}^{k}+G^{p}\left(x_{t}^{p}\right) v_{t}^{p} \\
x_{t+1}^{k} & =f^{k}\left(x_{t}^{p}\right)+F^{k}\left(x_{t}^{p}\right) x_{t}^{k}+G^{k}\left(x_{t}^{p}\right) v_{t}^{k} \\
y_{t} & =h\left(x_{t}^{p}\right)+H^{y}\left(x_{t}^{p}\right) x_{t}^{k}+e_{t},
\end{aligned}
$$

with process noise $v_{t}^{p} \sim \mathcal{N}\left(0, Q^{p}\right), v_{t}^{k} \sim \mathcal{N}\left(0, Q^{k}\right)$, and measurement noise $e_{t} \sim \mathcal{N}(0, R)$. It is assumed that these are all mutually independent white noise processes. Using (8) and (9), it is easy to verify that $p\left(x_{t+1}^{k} \mid x_{t}^{p}\right), p\left(x_{t+1}^{p} \mid x_{t}^{p}\right)$, and $p\left(y_{t} \mid x_{t}^{p}\right)$ are linear in $x_{t}^{k}$ and Gaussian.

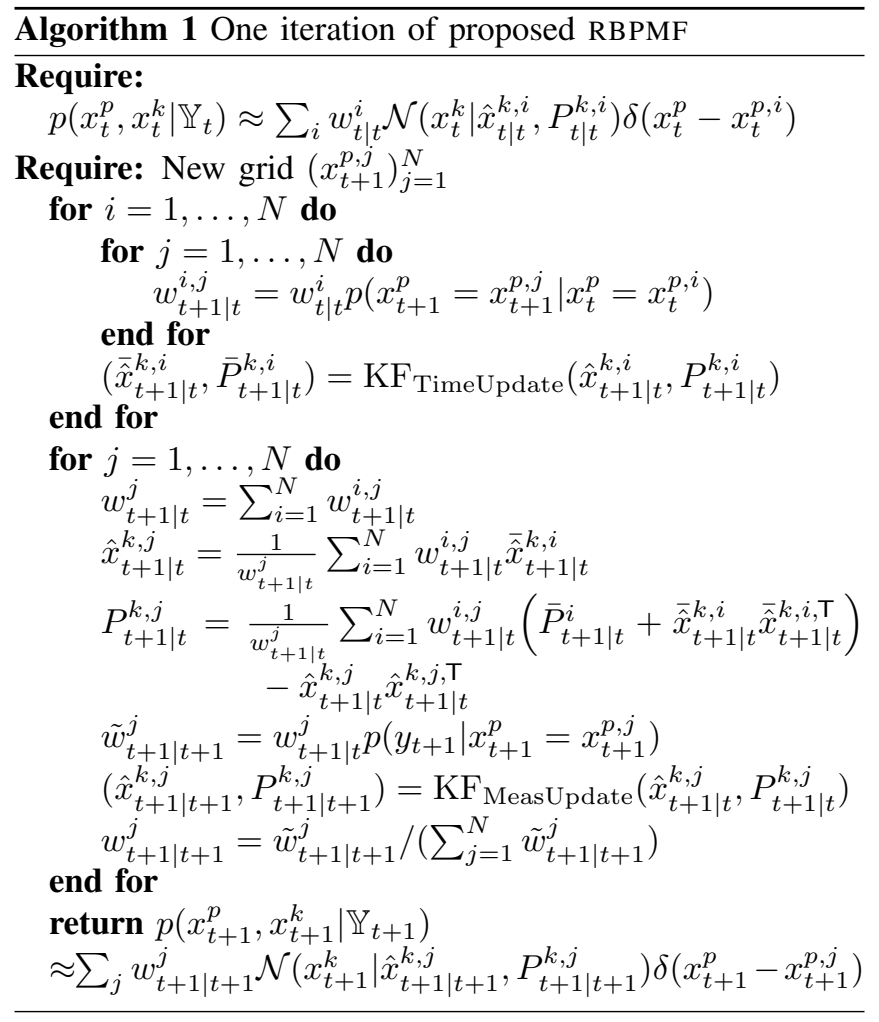

\section{Rao-Blackwellized Particle Filter}

The RBPF, sometimes denoted the marginalized particle filter (MPF), or mixture Kalman filters, [12-14] improves the performance and reduces the computational burden when a linear-Gaussian substructure is present. In (8) this means that KFs are used for the first part and a PF for the second part. Since the RBPF is used in comparison to the improved RBPMF in this paper, the reader is referred the detailed references above.

\section{Rao-Blackwellized Point-Mass Filter}

The RBPMF is a Bayesian filtering method originally put forth by [17], based on the Rao-Blackwellized marginal particle filter [18]. The basic idea is similar to the RBPF, however, the RBPMF deals with the marginal distribution $p\left(x_{t}^{p}, x_{t}^{k} \mid \mathbb{Y}_{t}\right)$ instead of the full posterior distribution of the discretized component, $p\left(\mathbb{X}_{t}^{p}, x_{t}^{k} \mid \mathbb{Y}_{t}\right)$. Hence, in contrast to (8), $p\left(x_{t}^{k} \mid x_{t}^{p}, \mathbb{Y}_{t}\right)$ is not conditionally linear-Gaussian. Thus, additional approximations are required. Assume that

$$
\begin{aligned}
p\left(x_{t} \mid \mathbb{Y}_{t}\right) & =p\left(x_{t}^{k} \mid x_{t}^{p}, \mathbb{Y}_{t}\right) p\left(x_{t}^{p} \mid \mathbb{Y}_{t}\right) \\
& \approx \sum_{i=1}^{N} w_{t \mid t}^{i} \mathcal{N}\left(x_{t}^{k} \mid \hat{x}_{t \mid t}^{k, i}, P_{t \mid t}^{k, i}\right) \delta\left(x_{t}^{p}-x_{t}^{p, i}\right),
\end{aligned}
$$

i.e., an existing conditionally linear-Gaussian approximation of $p\left(x_{t} \mid \mathbb{Y}_{t}\right)$ is available using $N$ grid points. Now, a method to compute $p\left(x_{t+1} \mid \mathbb{Y}_{t}\right)$ in the same form will be described. In order to compute $p\left(x_{t+1} \mid \mathbb{Y}_{t}\right)$, first define a grid $\left(x_{t+1}^{p, i}\right)_{j=1}^{n}$. Then, the joint distribution $p\left(x_{t+1}^{k}, x_{t+1}^{p}, x_{t}^{p} \mid \mathbb{Y}_{t}\right)$ can be computed from (10). Now marginalize $x_{t}^{p}$ away, 
TABLE I

$$
\begin{aligned}
& p\left(x_{t+1}^{k}, x_{t+1}^{p} \mid \mathbb{Y}_{t}\right) \\
& \approx \sum_{i, j=1}^{N} w_{t+1 \mid t}^{i, j} \mathcal{N}\left(x_{t+1}^{k} \mid \hat{x}_{t+1 \mid t}^{k, i, j}, P_{t+1 \mid t}^{k, i, j}\right) \delta\left(x_{t+1}^{p}-x_{t+1}^{p, j}\right),
\end{aligned}
$$

where a Gaussian distribution in $x_{t+1}^{k}$ is required to handle every combination of $x_{t}^{p, i}$ and $x_{t+1}^{p, j}[17,18]$. Thus $N^{2}$ KF means and covariance matrices must be saved and updated using $y_{t+1}$. Finally, the papers $[17,18]$ merge the means and covariance matrices using moment matching in order to close the recursion. In this paper, this is addressed somewhat differently, assuming that $p\left(x_{t+1}^{p} \mid x_{t}^{p}, x_{t}^{k}\right)=p\left(x_{t+1}^{p} \mid x_{t}^{p}\right)$, which is true for the state-space model presented in Sec. II-A. In that case, $\left(\hat{x}_{t+1 \mid t}^{k, i, j}, P_{t+1 \mid t}^{k, i, j}\right)$ coincide for every $j$, since $x_{t+1}^{p}$ does not provide any additional information about the linear state. It is then possible to directly merge the means and covariance matrices. Subsequently the measurement update can be performed identically as in a bootstrap RBPF. The method is given in Algorithm 1. Note that it is at no point necessary to store and update all $N^{2}$ means and covariance matrices, thus avoiding significant computational and storage cost. However, the algorithm has an overall complexity $\mathcal{O}\left(N^{2}\right)$ in general, as compared to the bootstrap RBPF's $\mathcal{O}(N)$.

\section{Application to Vehicle Speed Tracking}

In this section, the model and filtering algorithms described above are applied to a harmonics tracking problem using experimental accelerometer data from a passenger car. The estimated frequency will be compared to the ground truth as given by the wSS encoder.

\section{A. Experiments}

In order to verify the model and algorithms, they are applied to experimental data in the form of lateral accelerometer data collected from an in-vehicle sensor cluster in an Audi A6. A standard Vector logging device [25] samples the vehicle's FlexRay bus collecting accelerometer data from the ESP sensor cluster, as well as ground truth data in form of angular velocity from a WSS with a sampling rate of $100 \mathrm{~Hz}$. This sample rate was chosen to enable estimation of vehicle speed up to typical legal speed limits $(120 \mathrm{~km} / \mathrm{h})$, while limiting sample rate requirements and computational effort. The raw lateral accelerometer signal is high-pass filtered with a Butterworth filter of order 2 and cut-off frequency $8 \mathrm{~Hz}$ in order to remove the static level of the accelerometer signal as well as undesired resonance modes unrelated to the tracked harmonics, yielding the signal $y_{t}$.

\section{B. Rao-Blackwellization and Parametrization}

In order to apply the model (4) to the above algorithms, it must be put in a Rao-Blackwellized form (9). Letting $x^{p}=\omega$, and $x^{k}=\left[\alpha^{\top}, \beta^{\top}\right]^{\top}$, it is straightforward to see that this is possible, since conditional on $\omega$, (4c) and (4a) are linear-Gaussian. Note that $p\left(x_{t+1}^{p} \mid x_{t}^{p}, x_{t}^{k}\right)=p\left(x_{t+1}^{p} \mid x_{t}^{p}\right)$, so that the simplifying condition in Sec. III-D is satisfied. The parameter values used are given in Table I. The number

\begin{tabular}{|c|c|}
\hline Parameter & Value \\
\hline $\operatorname{var}\left(v_{t}^{\omega}\right)$ & 4 \\
\hline $\operatorname{var}\left(v_{t}^{\alpha^{(m)}}\right), \operatorname{var}\left(v_{t}^{\beta^{(m)}}\right)$ & 0.001 \\
\hline $\operatorname{var}\left(\alpha_{0}^{(m)}\right), \operatorname{var}\left(\beta_{0}^{(m)}\right)$ & 0.1 \\
\hline Tracked harmonics: $M$ & 3 \\
\hline Grid points, RBPMF: $N$ & 200 \\
\hline Particles, RBPF & 3000 \\
\hline
\end{tabular}

SELECTED FILTER PARAMETERS AND VALUES.

of grid points and particles were chosen in order to match the computational effort of the two algorithms, implemented in MATLAB $^{\circledR}$. The grid points in the RBPMF are fixed to a linear spacing on the interval $[10,120] \mathrm{rad} / \mathrm{s}$.

For the subharmonic summation, the number of samples used per batch was set to be 200 , i.e., the method will deliver a speed estimate every 2 seconds. Smaller batches yielded very low accuracy while larger ones were bad at handling changes in vehicle speed.

\section{Results and Discussion}

The data set used in this paper is of length $1000 \mathrm{~s}$. First, we focus on the interval $[220,270] \mathrm{s}$, where an illustration of the spectrum is also available in Fig. 2, to illustrate the typical transient performance of the methods. In the left part of Fig. 3, the RBPMF is depicted, together with the confidence interval and the ground truth from the WSS signal; similarly, in the right part, the tracking results for two realizations of the bootstrap RBPF with multinomial resampling are shown. The realizations were selected as the 4 th best and 4 th worst with respect to RMSE over 15 Monte Carlo runs of the RBPF using the same data.

As can be seen, the RBPMF robustly captures the fundamental frequency in less than $2 \mathrm{~s}$. The confidence interval indicates that the RBPMF considers half the correct fundamental frequency as a possibility, but discards it after a few seconds. In comparison, one of the realizations of the $\mathrm{RBPF}$ is unable to capture the correct mode and instead considers the true first harmonic as the second harmonic. Since it fails to capture any other harmonics, its uncertainty is also higher. When the first harmonic loses energy, after $t=$ 260 (see Fig. 2), its confidence interval is large, indicating an uncertain estimate. The other realization of the RBPF manages to capture the true mode but has a higher RMSE than the RBPMF, as can be seen in Table II.

In Fig. 4, the RBPMF and SHS algorithms are illustrated for a longer time interval of 15 minutes. The RBPMF correctly tracks the true angular velocity for the full test drive. The

TABLE II

RMSE OF DIFFERENT ALGORITHMS FROM TIME 220 TO 270 WHEN THE FILTERS WERE INITIALIZED WITH UNKNOWN INITIAL ANGULAR VELOCITY.

\begin{tabular}{lc}
\hline & RMSE \\
\hline RBPMF & 2.66 \\
RBPF & 3.28
\end{tabular}



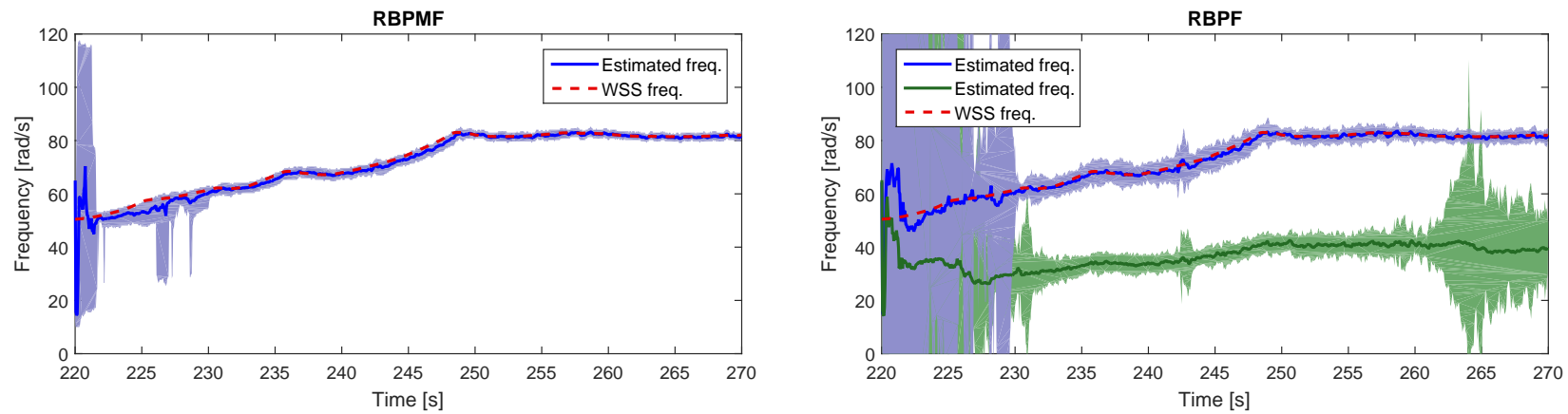

Fig. 3. Estimated fundamental frequency from the RBPMF and two realizations of the RBPF compared to ground truth (measured wSS signal). The 98\% confidence intervals have lighter shades. This illustrates the transient behavior of the algorithms when initial angular velocity is unknown. Note that the deterministic RBPMF accurately captures the fundamental frequency, while one of the stochastic RBPF realizations misses it by a factor of 2 due to insufficient particle representation on the support of the posterior. The filters approximate the full non-Gaussian posterior state distribution; thus, the confidence intervals are asymmetric with respect to the posterior mean.

SHS method is often able to detect the correct fundamental frequency, however it also sometimes misses it. When the velocity changes quickly, e.g., $t=800 \mathrm{~s}$, this can be particularly problematic. In this case SHS only manages to capture one correct sample in the upper half of the transient. Even if the detections were to be input into a tracker [26], there would be a possibility of losing track at that point.

To numerically compare the RBPMF or RBPF with the SHS, we favor the latter by only considering the detections which were within a certain distance $(5 \mathrm{rad} / \mathrm{s})$ from the true angular velocity. In addition, we correct for missed detections due to detecting half the true fundamental frequency. Since the SHS algorithm computes an estimate over a period of 2 seconds, we compare its speed estimate with the mean of the RBPMF and RBPF estimate over the same period of time when computing the RMSE. The RBPF, when it captures the correct track, has a similar behavior as the RBPMF in stationarity on this particular signal, thus it is not illustrated. In the full data sequence in Fig. 4, the RMSE of the various algorithms can be seen in Table III, where the RBPMF slightly outperforms the RBPF, and significantly outperforms the SHS.

There are multiple differences between the algorithms which cause these differences. The resampling in the RBPF increases the variance of the estimate and may discard potentially correct solutions. A particle representation will not necessarily represent a multi-modal distributions in a robust manner. In contrast, the RBPMF will consider all frequencies in the considered region, not only the most likely ones at the moment. This is an advantage emphasized in [17], and indeed it applies here as well.

TABLE III

RMSE OF DIFFERENT ALGORITHMS FROM FOR THE 15 MINUTE LONG INTERVAL WHEN THE FILTERS WERE INITIALIZED WITH THE TRUE ANGULAR VELOCITY.

\begin{tabular}{ll}
\hline & RMSE \\
\hline RBPMF & 0.325 \\
RBPF & 0.343 \\
SHS & 0.519
\end{tabular}

The SHS algorithm discards the phase spectrum and does not exploit the continuous nature of the signal in a strict manner, although we discard incorrect detections before computing the error in the evaluation above. Nor does its batch processing consider that the frequency is time-varying.

\section{Conclusions}

In the paper a novel approach to estimate the wheel velocity of a vehicle based on harmonics in collected accelerometer measurements has been presented. An extension and simplification of the Rao-Blackwellized point mass filter (RBPMF) was presented for a particular model class, reducing the computational burden significantly. Two different parametric solutions utilizing Bayesian filtering (RBPMF, RBPF) and the subharmonic summation (SHS) method based on spectrum maximization were presented and verified on experimental data collected from an Audi A6. The concept of tracking vehicle speed through chassis vibrations is shown to be feasible, and the proposed RBPMF variant appears superior in robustness and performance to the RBPF and SHS since it can handle the fundamentally multi-modal harmonics tracking problem in a better manner.

\section{ACKNOWLEDGMENTS}

This work was partially supported by the Wallenberg Autonomous Systems Program (WASP). NIRA Dynamics AB provided the experimental data.

\section{REFERENCES}

[1] F. Gustafsson, "Rotational speed sensors: Limitations, preprocessing and automotive applications." IEEE Instrumentation \& Measurement Magazine, vol. 13, no. 2, p. 16, 2010.

[2] J. Zhang, K. Zhang, R. Grenfell, and R. Deakin, "Short note: On the relativistic doppler effect for precise velocity determination using GPS," Journal of Geodesy, vol. 80, no. 2, pp. 104-110, 2006.

[3] S. Zair, S. Le Hegarat-Mascle, and E. Seignez, "Coupling outlier detection with particle filter for GPS-based localization," in Proceedings of the IEEE 18th International Conference on Intelligent Transportation Systems (ITSC), Las Palmas, Spain, Sep. 2015.

[4] Z. Syed, P. Aggarwal, N. Xiaoji, and N. El-Sheimy, "Civilian vehicle navigation: required alignment of the inertial sensors 

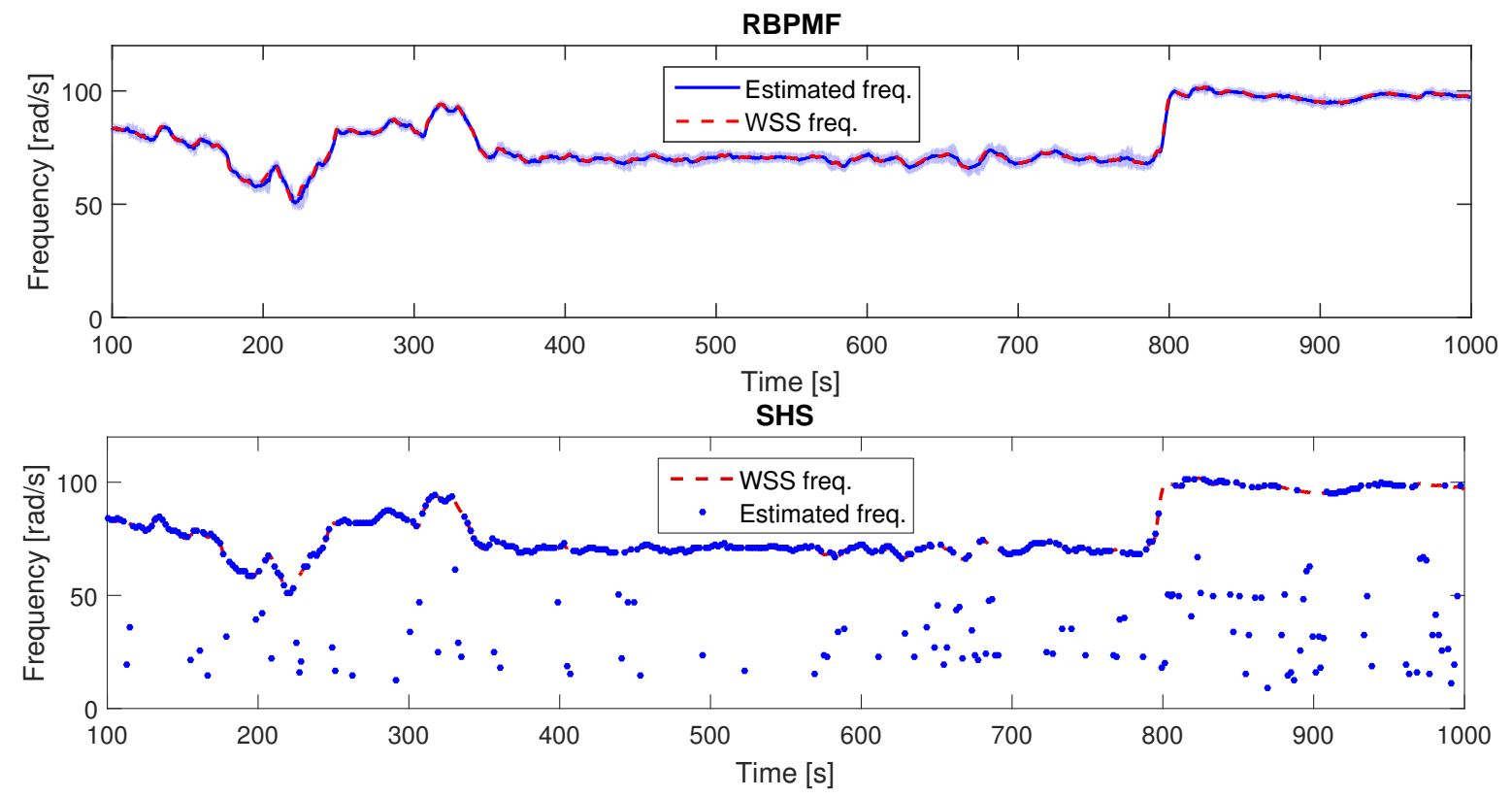

Fig. 4. Estimated fundamental frequency from the RBPMF and SHS compared to ground truth (measured wSS signal). This illustrates the stationary behavior of the algorithms. For the RBPMF, the $98 \%$ confidence intervals has lighter shades.

for acceptable navigation accuracies." IEEE Transactions on Vehicular Technology, vol. 57, no. 6, pp. 3402 - 3412, 2008.

[5] C. Vicek, P. McLain, and M. Murphy, "GPS/dead reckoning for vehicle tracking in the "urban canyon" environment." in Proceedings of the IEEE-IEE Vehicle Navigation and Information Systems Conference, Ottawa, Canada, Oct. 2015, pp. A34-A41.

[6] R. Kumar, "Optimum filters and smoothers design for carrier phase and frequency tracking," NASA Jet Propulsion Laboratory, Tech. Rep., 1987, JPL publication 87-10.

[7] P. J. Parker and B. D. Anderson, "Frequency tracking of nonsinusoidal periodic signals in noise," Signal Processing, no. 20, pp. $127-152,1990$.

[8] B. La Scala and R. Bitmead, "Design of an extended Kalman filter frequency tracker." IEEE Transactions on Signal Processing, vol. 44, no. 3, pp. 739 - 742, 1996.

[9] W. Ng, J. Chunlin, W.-K. Ma, and H. C. So, "A study on particle filters for single-tone frequency tracking," IEEE Transactions on Aerospace and Electronic Systems, vol. 45, no. 3, pp. 1111 - 1125, 2009.

[10] C. Dubois and M. Davy, "Joint detection and tracking of timevarying harmonic components: a flexible Bayesian approach." IEEE Transactions on Audio, Speech and Language Processing, vol. 15, no. 4, pp. 1283 - 1295, 2007.

[11] S. Kim, L. Holmstrom, and J. McNames, "Multiharmonic tracking using marginalized particle filters." in Proceedings of the 30th Annual International Conference of the IEEE Engineering in Medicine and Biology Society, Vancouver, Canada, Aug. 2008, pp. 29-33.

[12] G. Casella and C. P. Robert, "Rao-Blackwellisation of sampling schemes," Biometrika, vol. 83, no. 1, pp. 81-94, 1996.

[13] T. Schön, F. Gustafsson, and P.-J. Nordlund, "Marginalized particle filters for mixed linear / nonlinear state-space models," IEEE Transactions on Signal Processing, vol. 53, no. 7, pp. 2279-2289, Jul. 2005.

[14] T. B. Schön, R. Karlsson, and F. Gustafsson, "The marginalized particle filter in practice," in Proceedings of IEEE Aerospace Conference, Big Sky, MT, USA, Mar. 2006.

[15] D. Hermes, "Measurement of pitch by subharmonic summa- tion." Journal of the Acoustical Society of America, vol. 83, no. 1 , pp. 257-264, 1988.

[16] A. de Cheveigne and H. Kawahara, "YIN, a fundamental frequency estimator for speech and music." Journal of the Acoustical Society of America, vol. 111, no. 4, pp. 1917 1930, 2002.

[17] V. Smidl and M. Gasperin, "Rao-Blackwellized point mass filter for reliable state estimation." in Proceedings of 16th IEEE International Conference on Information Fusion, Istanbul, Turkey, Jul. 9-12 2013, pp. 312-318.

[18] F. Lindsten, "Rao-Blackwellised particle methods for inference and identification." Licentiate Thesis No 1480, Department of Electrical Engineering, Linköpings universitet, Sweden, 2011.

[19] N. J. Gordon, D. J. Salmond, and A. F. M. Smith, "Novel approach to nonlinear/non-Gaussian Bayesian state estimation," IEE Proceedings-F Radar and Signal Processing, vol. 140, no. 2, pp. 107-113, Apr. 1993.

[20] A. Doucet, N. de Freitas, and N. Gordon, Eds., Sequential Monte Carlo Methods in Practice, ser. Statistics for Engineering and Information Science. New York: Springer-Verlag, 2001.

[21] R. E. Kalman, "A new approach to linear filtering and prediction problems," Transactions of the American Society of Mechanical Engineering - Journal Basic Engieering, Series $D$, vol. 82, pp. 35-45, Mar. 1960.

[22] T. Kailath, A. H. Sayed, and B. Hassibi, Linear Estimation. Prentice-Hall, Inc, 2000.

[23] R. S. Bucy and K. D. Senne, "Digital synthesis of non-linear filters," Automatica, vol. 7, no. 3, pp. 287-298, 1971.

[24] Z. Chen, "Bayesian filtering: From Kalman filters to particle filters, and beyond," Statistics, vol. 182, no. 1, pp. 1-69, 2003.

[25] Vector Informatik GmbH, Manual, VN8900 Interface Family.

[26] S. S. Blackman and R. Popoli, Design and analysis of modern tracking systems, ser. Artech House radar library. Artech House, Inc, 1999. 